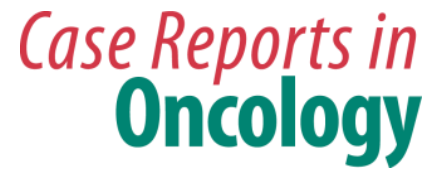

Case Rep Oncol 2018;11:549-556

DOI: 10.1159/000491862

Published online: August 17, 2018

2018 The Author(s)

Published by S. Karger AG, Basel

www.karger.com/cro

This article is licensed under the Creative Commons Attribution-NonCommercial 4.0 International License (CC BY-NC) (http://www.karger.com/Services/OpenAccessLicense) Usage and distribution for commercial purposes requires written permission.

\title{
Severe Gastritis after Administration of Nivolumab and Ipilimumab
}

\author{
Yoshito Nishimura $^{a}$ Miho Yasuda $^{a}$ Kazuki Ocho ${ }^{a}$ Masaya Iwamuro ${ }^{b}$ \\ Osamu Yamasakic ${ }^{c}$ Takehiro Tanaka $^{d}$ Fumio Otsuka ${ }^{a}$ \\ aDepartment of General Medicine, Okayama University Graduate School of Medicine, \\ Dentistry, and Pharmaceutical Sciences, Okayama, Japan; ${ }^{b}$ Department of \\ Gastroenterology and Hepatology, Okayama University Graduate School of Medicine, \\ Dentistry, and Pharmaceutical Sciences, Okayama, Japan; 'Department of Dermatology, \\ Okayama University Graduate School of Medicine, Dentistry, and Pharmaceutical \\ Sciences, Okayama, Japan; ${ }^{\mathrm{d} D e p a r t m e n t}$ of Pathology, Okayama University Graduate \\ School of Medicine, Dentistry, and Pharmaceutical Sciences, Okayama, Japan
}

\section{Keywords}

Immune checkpoint inhibitor · Ipilimumab · Nivolumab · Helicobacter pylori · Gastritis

\begin{abstract}
Immune checkpoint inhibitors such as ipilimumab, a cytotoxic T-lymphocyte-associated antigen-4 inhibitor, have been widely used for advanced malignancies. As these inhibitors improve antitumor immunity via T-cell modulation, immune-mediated adverse events associated with T-cell activation, such as colitis, might occur. Herein, we describe a 75-year-old Japanese woman with metastatic malignant melanoma who developed hemorrhagic gastritis after ipilimumab treatment. There was no macroscopic or clinical improvement of gastritis after proton pump inhibitor treatment. However, her condition improved after approximately 3 weeks of corticosteroid therapy and Helicobacter pylori eradication. This case suggests a potential association between severe gastritis and immune checkpoint inhibitor treatment. Although several reports have mentioned ipilimumab-associated colitis, gastritis is considered to be rare. In the
\end{abstract}

KARGER
Dr. Yoshito Nishimura

Department of General Medicine

Okayama University Graduate School of Medicine, Dentistry, and Pharmaceutical

Sciences

2-5-1 Shikata-cho, Kita-ku, Okayama 700-8558 (Japan)

E-Mailme421060@s.okayama-u.ac.jp 


\section{Case Reports in Oncology}

Case Rep Oncol 2018;11:549-556

DOI: $10.1159 / 000491862$

(C) 2018 The Author(s). Published by S. Karger AG, Base www.karger.com/cro

Nishimura et al.: Severe Gastritis after Administration of Nivolumab and Ipilimumab

present case, $H$. pylori-associated gastritis might have been exacerbated by the T-cell modulation effect of ipilimumab. To date, no report has clarified the mechanism by which ipilimumab modifies $H$. pylori infection. The present treatment course provides a helpful perspective for similar cases.

(C) 2018 The Author(s)

Published by S. Karger AG, Basel

\section{Introduction}

Immune checkpoint inhibitors, such as ipilimumab, are novel chemotherapeutic agents for various metastatic malignancies. Ipilimumab is a completely humanized monoclonal antibody which enhances antitumor immunity through the attenuation of negative signaling from cytotoxic T-lymphocyte-associated antigen-4 (CTLA-4) on T cells. However, CTLA-4 inhibition can cause immune-mediated adverse events, such as colitis [1]. Although colitis has been extensively reported as one of the most common adverse events [1], detailed clinical history and esophagogastroduodenoscopy (EGD) findings of gastritis after ipilimumab administration have not yet been reported. Herein, we present the case of a female patient with metastatic malignant melanoma who developed gastritis after ipilimumab administration. She also had Helicobacter pylori infection, which might have contributed to her gastritis. Considering the possible autoimmune features of ipilimumab-associated gastritis, corticosteroids might be the most appropriate first-line treatment, as in the case of colitis. Our patient received corticosteroids and $H$. pylori eradication therapy, and her gastritis improved after treatment. It is unclear whether ipilimumab exacerbates preexisting gastric inflammation. Additionally, it is important to clarify whether there is a causal relationship between $H$. pylori treatment and clinical improvement. In this report, we extensively discuss the possible relationships among gastritis, immune checkpoint inhibitors, and H. pylori infection.

\section{Case Report}

A 75-year-old Japanese woman with metastatic malignant melanoma was admitted to the hospital for epigastric pain and loss of appetite. She was previously treated with nivolumab at the Department of Dermatology 2 months before admission. However, nivolumab was stopped because of the exacerbation of metastatic liver lesions, and she was administered ipilimumab once at a dose of $3 \mathrm{mg} / \mathrm{kg} 2$ weeks before admission. She complained of appetite loss, nausea, and vomiting 7 days after ipilimumab administration. She visited her dermatologist because of persistent symptoms 1 day before admission, and she was referred to the Department of General Medicine for further evaluation and treatment. During examination, no diarrhea, hematochezia, or abdominal pain was noted, and her medical history was otherwise unremarkable. On admission, her C-reactive protein level was elevated $(2.46 \mathrm{mg} / \mathrm{dL})$, without an increase in the white blood cell count (Table 1). A computed tomography (CT) scan without contrast revealed diffuse edematous gastric wall thickening, and subsequent EGD demonstrated diffusely erythematous and edematous gastric mucosa without apparent ulcers (Fig. 1a, b). The gastric mucosa was covered with a whitish, fibrin-like membrane. Magnified observation of the gastric body with narrow-band imaging revealed destruction of the 


\section{Case Reports in Oncology}

Case Rep Oncol 2018;11:549-556

DOI: $10.1159 / 000491862$

(c)

(C) 2018 The Author(s). Published by S. Karger AG, Basel www.karger.com/cro

Nishimura et al.: Severe Gastritis after Administration of Nivolumab and Ipilimumab

glandular structure of the surface (Fig. 1c). Multiple round mucosae were seen in the gastric antrum (Fig. 1d), and they were more prominent after indigo carmine spraying (Fig. 1e). Magnified observation with narrow-band imaging (Fig. 1f) showed diffusely denuded areas of the mucosa and residual mucosal islands, where the glandular structure was relatively intact (arrows). Oozing hemorrhages were noted with air infusion, suggesting mucosal friability (Fig. 1a, arrow). Pathological analysis of the whitish membrane that adhered to the gastric mucosa showed fibrin and numerous polynuclear leucocytes (Fig. 2a). Biopsy of the gastric body revealed inflammatory cell infiltration into the lamina propria, destruction of ducts, and erosive mucosa (Fig. 2b). Although no evidence of malignancy was noted in the biopsy specimen, H. pylori was detected.

Drug-induced gastritis was suspected, and thus the patient was administered an intravenous proton pump inhibitor only per os. Follow-up EGD performed 6 days after admission demonstrated large mucosal defects in the duodenal bulb, where duodenal villi were diffusely damaged (Fig. 2c, d). Additionally, significant hemorrhage was noted after air insufflation during EGD (Fig. 2e). Thus, the gastroduodenal lesions were considered to have deteriorated. Based on these findings, she was diagnosed with grade 3 drug-induced gastroduodenitis and received intravenous prednisone $(1.0 \mathrm{mg} / \mathrm{kg} /$ day $)$ along with a proton pump inhibitor. Additionally, she received amoxicillin, clarithromycin, and a proton pump inhibitor (triple therapy) for H. pylori infection. On the day of her diagnosis, increased amylase $(390 \mathrm{U} / \mathrm{L}$; reference range 40-132 U/L) and lipase (929 U/L; reference range 40-150 U/L) levels were noted (Table 1). A second CT scan without contrast enhancement showed enlargement of the pancreas without peripancreatic fat stranding or fluid collection. Drug-induced pancreatitis was suspected, and prednisone treatment was continued. Another EGD performed 20 days after admission (15 days after steroid therapy) revealed slight improvement in mucosal erythema and patchy appearance of mucosal regeneration (Fig. 3a-e). A gastric biopsy specimen showed slight improvement in inflammation with granulation tissue and intestinal metaplasia. Additionally, lipase and amylase levels had decreased to 188 and $224 \mathrm{U} / \mathrm{L}$, respectively (Table 1). Considering improved endoscopic findings, prednisone was tapered. She was discharged 23 days after admission, and she is being followed up on an outpatient basis.

\section{Discussion}

Immune checkpoint inhibitors are novel therapeutic options for malignant tumors. However, their use is associated with broad immunological adverse events, such as enterocolitis, hypophysitis, and pancreatitis [1-4]. Notably, enterocolitis has been reported in up to approximately $8 \%$ of patients who have received ipilimumab [5]. In contrast, nivolumab-induced colitis is rare, and only 2 cases have been reported to date [6, 7]. As ipilimumab is a monoclonal antibody against CTLA-4, which negatively regulates T-cell activation, ipilimumab-induced colitis might result from an increase in cytotoxic CD8+ $\mathrm{T}$ cells relative to regulatory FoxP3+ T cells in the colon [8].

Although ipilimumab-induced colitis is a common adverse event, ipilimumab-induced gastritis is rare [9]. Recently, Boike et al. [10] reported a case of nivolumab-associated esophagitis and gastritis. Their patient was a 93-year-old woman with Hodgkin's lymphoma who had a 2-month history of dysphagia and diarrhea. She was administered nivolumab 6 months 


\section{Case Reports in Oncology}

Case Rep Oncol 2018;11:549-556

DOI: $10.1159 / 000491862$

(C) 2018 The Author(s). Published by S. Karger AG, Base www.karger.com/cro

Nishimura et al.: Severe Gastritis after Administration of Nivolumab and Ipilimumab

before symptom onset. She showed significant uptake in the stomach on positron emission tomography-CT (PET-CT) and edematous gastric mucosa with diffuse erythema on EGD. She was eventually diagnosed with nivolumab-induced gastritis, and her condition improved with intravenous prednisone ( $1 \mathrm{mg} / \mathrm{kg} /$ day). Although we did not perform PET-CT, our patient had similar EGD findings and a favorable treatment response. As the pathophysiology of ipilimumab-induced colitis is associated with augmented T-cell responses in the intestine, we speculate that ipilimumab-induced gastritis is also associated with T-cell activation in the gastric mucosa after drug administration. Additionally, our patient had $H$. pylori infection, which might have contributed to her gastritis. Anderson et al. [11] reported the importance of CTLA4 for establishing T-cell anergy in an in vivo model of $H$. pylori infection in mice that received anti-CTLA-4 Fab fragments. The researchers concluded that anti-CTLA-4 antibodies could induce extensive gastric inflammation in $H$. pylori-infected individuals. Conversely, Watanabe et al. [12] suggested that anti-CTLA-4 antibodies might suppress the inflammatory immune response in $H$. pylori-associated gastritis. Further investigations are needed to determine whether ipilimumab administration is associated with an increased immune response to H. pylori.

With regard to ipilimumab-induced colitis treatment, guidelines mentioning specific management and treatment plans are available. The FDA recommends ipilimumab discontinuation and corticosteroid administration (1-2 mg/kg/day) in cases of severe colitis. Infliximab, an anti-TNF $\alpha$ monoclonal antibody, might be effective for corticosteroid-refractory colitis [13]. In the present case of gastritis, a corticosteroid $(1 \mathrm{mg} / \mathrm{kg} /$ day) was administered, which is similar to the approach for ipilimumab-induced colitis. Considering the possibility that ipilimumab worsened the $H$. pylori-associated gastritis, our patient was treated with triple therapy to eradicate the $H$. pylori infection. After treatment, her gastritis showed both gross and pathological remission. Although we could not confirm whether the corticosteroid treatment and/or H. pylori eradication treatment had contributed to disease remission, the present treatment course provides a helpful perspective for similar cases.

\section{Conclusions}

We present a case of gastritis that occurred after ipilimumab administration. Gastritis is considered a rare complication of ipilimumab treatment, and it might involve T-cell modulation and autoimmune features. The clinical course might be more complicated when there is preexisting $H$. pylori infection. Early diagnosis and appropriate treatment, including corticosteroid therapy and $H$. pylori eradication therapy, are crucial. Although the possible pathophysiological mechanisms of gastritis have been speculated on in patients with previous ipilimumab administration, the significance of concomitant $H$. pylori infection is yet to be determined.

\section{Statement of Ethics}

The authors have no ethical conflicts to report. Informed consent was obtained from the patient for the publication of this case report and any accompanying images. 


\section{Disclosure Statement}

The authors have no conflicts of interest to report.

\section{References}

1 O'Connor A, Marples M, Mulatero C, Hamlin J, Ford AC. Ipilimumab-induced colitis: experience from a tertiary referral center. Therap Adv Gastroenterol. 2016 Jul;9(4):457-62.

2 Messmer M, Upreti S, Tarabishy Y, Mazumder N, Chowdhury R, Yarchoan M, et al. Ipilimumab-induced enteritis without colitis: a new challenge. Case Rep Oncol. 2016 Nov;9(3):705-13.

3 Brilli L, Danielli R, Ciuoli C, Calabrò L, Di Giacomo AM, Cerase A, et al. Prevalence of hypophysitis in a cohort of patients with metastatic melanoma and prostate cancer treated with ipilimumab. Endocrine. 2017 Dec;58(3):535-41.

4 Friedman CF, Clark V, Raikhel AV, Barz T, Shoushtari AN, Momtaz P, et al. Thinking critically about classifying adverse events: incidence of pancreatitis in patients treated with nivolumab + ipilimumab. J Natl Cancer Inst. 2016 Dec;109(4):djw260.

5 Rastogi P, Sultan M, Charabaty AJ, Atkins MB, Mattar MC. Ipilimumab associated colitis: an IpiColitis case series at MedStar Georgetown University Hospital. World J Gastroenterol. 2015 Apr;21(14):4373-8.

6 Kubo K, Kato M, Mabe K. Nivolumab-associated colitis mimicking ulcerative colitis. Clin Gastroenterol Hepatol. 2017 Sep;15(9):A35-6.

7 Yanai S, Nakamura S, Matsumoto T. Nivolumab-induced colitis treated by infliximab. Clin Gastroenterol Hepatol. 2017 Apr;15(4):e80-1.

8 Arriola E, Wheater M, Lopez MA, Thomas G, Ottensmeier C. Evaluation of immune infiltration in the colonic mucosa of patients with ipilimumab-related colitis. Oncoimmunology. 2016 Jul;5(9):e1209615.

9 Beck KE, Blansfield JA, Tran KQ, Feldman AL, Hughes MS, Royal RE, et al. Enterocolitis in patients with cancer after antibody blockade of cytotoxic T-lymphocyte-associated antigen 4. J Clin Oncol. 2006 May;24(15):2283-9.

10 Boike J, Dejulio T. Severe esophagitis and gastritis from nivolumab therapy. ACG Case Rep J. 2017 Apr;4:e57.

11 Anderson KM, Czinn SJ, Redline RW, Blanchard TG. Induction of CTLA-4-mediated anergy contributes to persistent colonization in the murine model of gastric Helicobacter pylori infection. J Immunol. 2006 May;176(9):5306-13.

12 Watanabe K, Murakami K, Sato R, Okimoto T, Maeda K, Nasu M, et al. CTLA-4 blockade inhibits induction of Helicobacter pylori-associated gastritis in mice. Clin Exp Immunol. 2004 Jan;135(1):29-34.

13 Beniwal-Patel P, Matkowskyj K, Caldera F. Infliximab therapy for corticosteroid-resistant ipilimumabinduced colitis. J Gastrointestin Liver Dis. 2015 Sep;24(3):274. 


\section{Case Reports in Oncology}
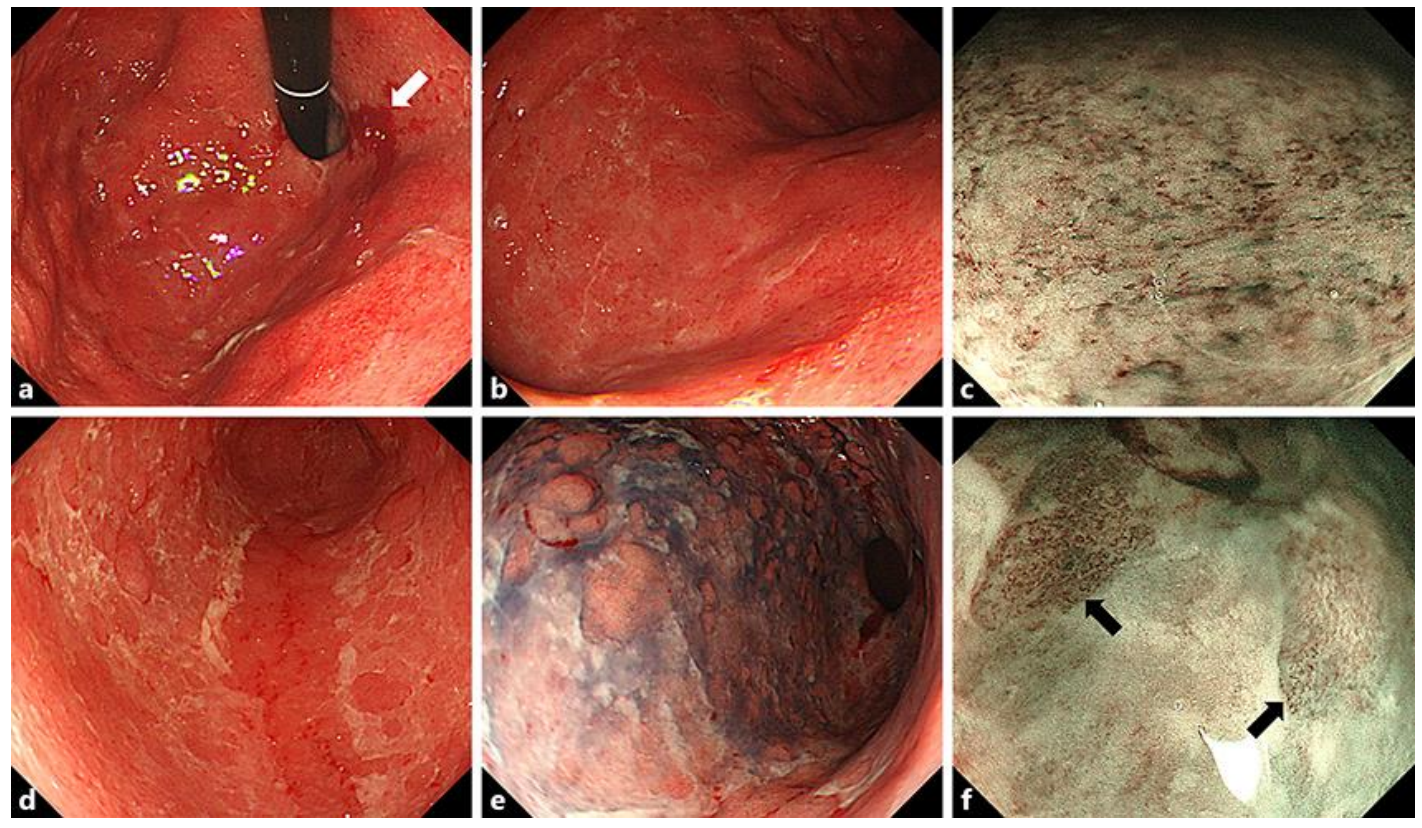

Fig. 1. EGD on admission. $\mathbf{a}, \mathbf{b}$ The stomach was diffusely erythematous and edematous. Despite the absence of ulcers, oozing hemorrhages with air infusion were seen. These findings were consistent with a fragile gastric mucosa (a: gastric body; b: antrum). c Magnifying observation of the gastric body with narrow-band imaging revealed destruction of the glandular structure of the surface. $\mathbf{d}$ In the gastric antrum, multiple round-shaped mucosae were seen. $\mathbf{e}$ The mucosal lesions were emphasized with indigo carmine spraying. f Narrow-band imaging revealed diffusely denuded areas of mucosa and residual mucosal islands, where the glandular structure was relatively intact (arrows). EGD, esophagogastroduodenoscopy. 


\section{Case Reports in Oncology}
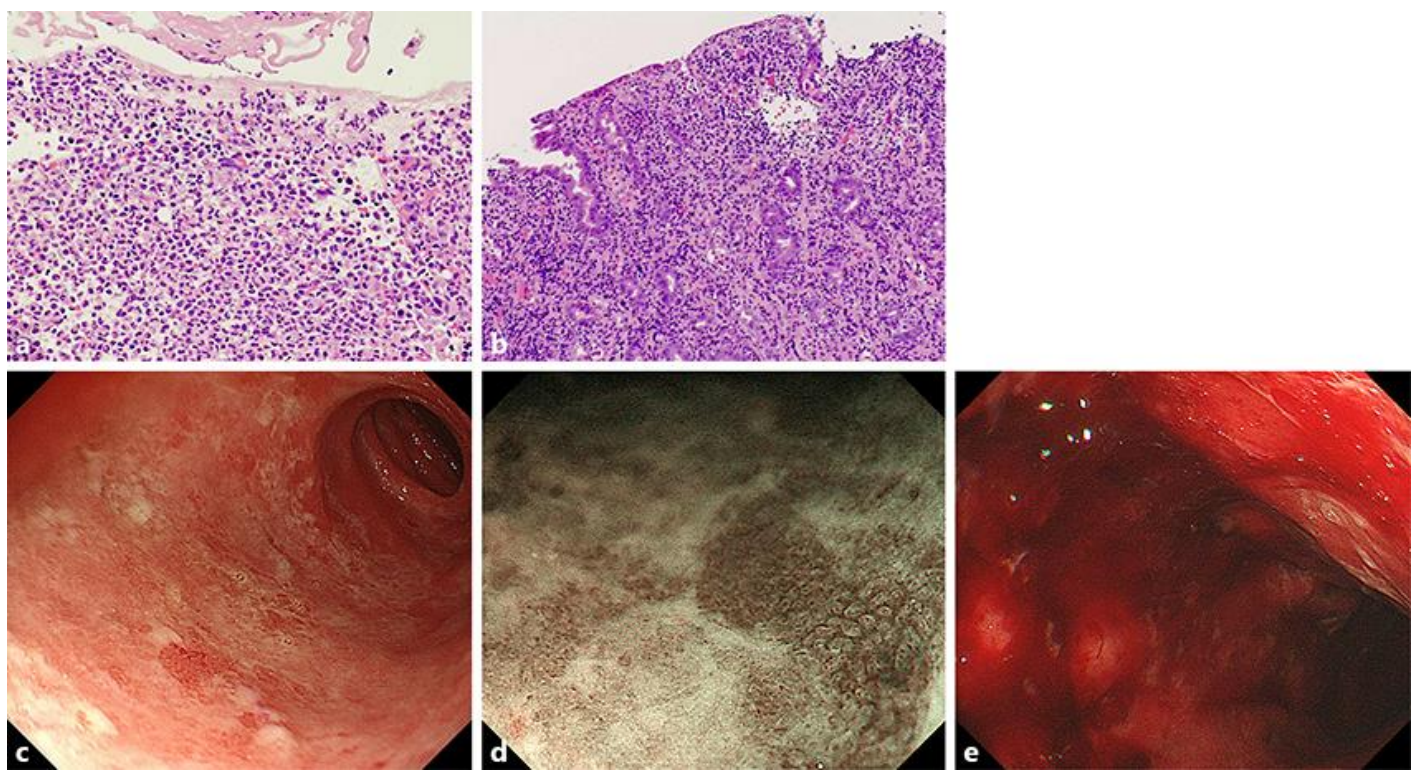

Fig. 2. a Pathological findings of the gastric mucosa on admission and follow-up EGD before prednisone treatment. Magnification $\times 100$. $\mathbf{b}$ Gastric biopsy showed total glandular atrophy and marked infiltration of lymphocytes and neutrophils in the lamina propria. Magnification $\times 400$. c-e Follow-up EGD showed that a proton pump inhibitor alone had not improved the macroscopic findings of gastritis. Large mucosal defects and significant erythema with hemorrhage were noted. EGD, esophagogastroduodenoscopy. 


\section{Case Reports in Oncology}

\begin{tabular}{l|l}
\hline Case Rep Oncol 2018;11:549-556 \\
\hline DOI: 10.1159/000491862 & $\begin{array}{l}\text { (c) 2018 The Author(s). Published by S. Karger AG, Basel } \\
\text { www.karger.com/cro }\end{array}$ \\
\hline
\end{tabular}

Nishimura et al.: Severe Gastritis after Administration of Nivolumab and Ipilimumab

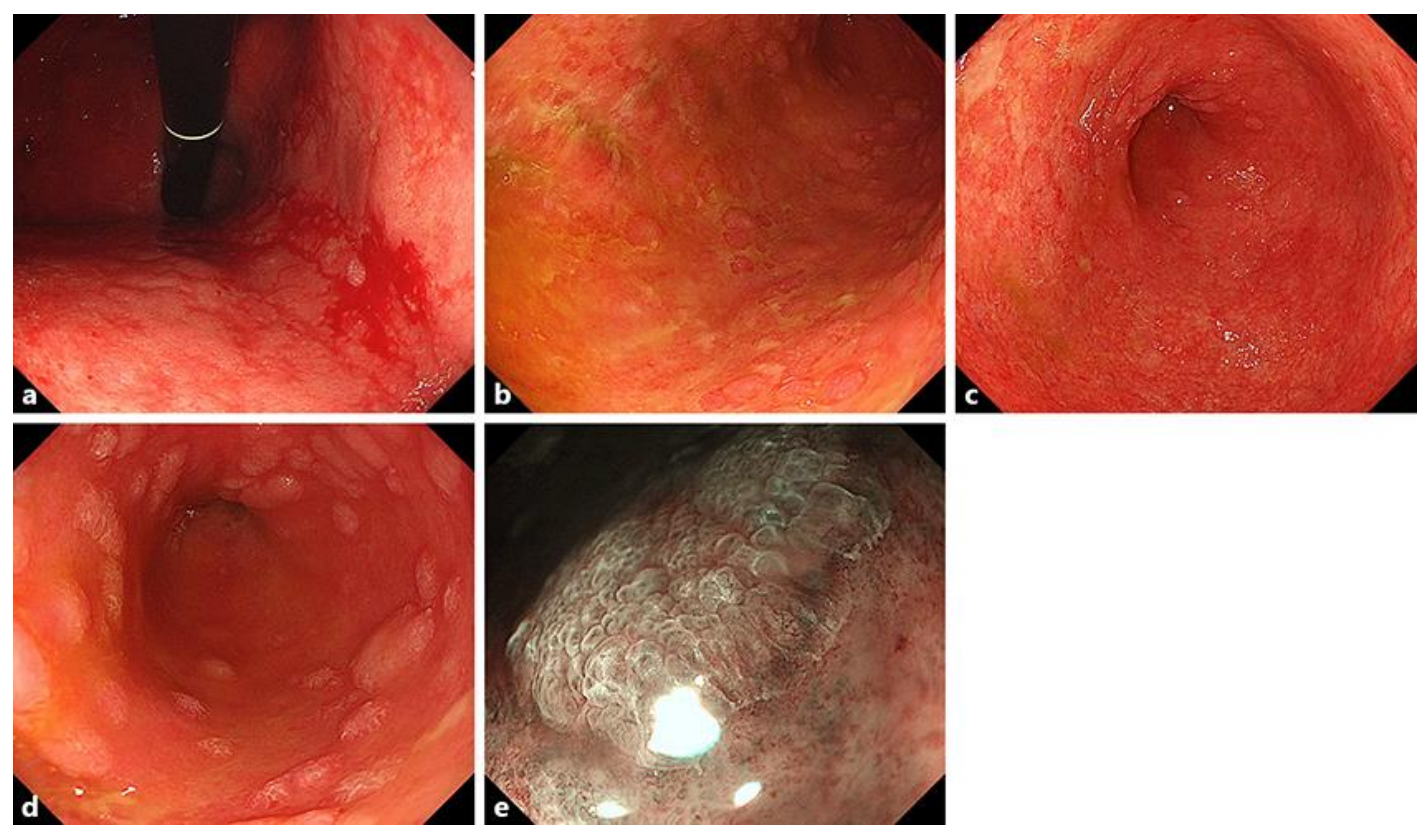

Fig. 3. EGD after prednisone treatment. Erythema severity showed improvement when compared with the previous findings. Regenerating mucosa was observed mainly in the antral area. EGD, esophagogastroduodenoscopy.

Table 1. Laboratory data

\begin{tabular}{lllll}
\hline Variable & $\begin{array}{l}\text { Reference } \\
\text { range }\end{array}$ & $\begin{array}{l}\text { Value on } \\
\text { admission }\end{array}$ & $\begin{array}{l}\text { Value on } \\
\text { day } 6\end{array}$ & $\begin{array}{l}\text { Value on } \\
\text { day 21 }\end{array}$ \\
\hline Blood & & & & 44.6 \\
Hematocrit, \% & $39.0-52.0$ & 37.9 & 38.3 & 14.8 \\
Hemoglobin, \% & $13.6-17.0$ & 13.1 & 12.8 & 6,620 \\
White blood cell count per mm ${ }^{3}$ & $3,500-8,500$ & 3,270 & 3,990 & $270 \times 10^{3}$ \\
Platelet count per mm ${ }^{3}$ & $130-300 \times 10^{3}$ & $243 \times 10^{3}$ & $276 \times 10^{3}$ & 129 \\
Sodium, mEq/L & $135-147$ & 134 & 133 & 4.7 \\
Potassium, mEq/L & $3.6-5.0$ & 4.1 & 3.8 & 95 \\
Chloride, mEq/L & $96-110$ & 102 & 101 & 22.7 \\
Blood urea nitrogen, mg/dL & $9.0-20.0$ & 12.3 & 7.5 & 0.64 \\
Creatinine, mg/dL & $0.40-1.10$ & 0.58 & 0.48 & 188 \\
Amylase, U/L & $40-132$ & & 390 & 224 \\
Lipase, U/L & $40-150$ & & 929 & 0.54 \\
C-reactive protein, mg/dL & $0.00-0.40$ & 2.46 & 4.33 & \\
Cytomegalovirus antigenemia & negative & negative & & \\
\hline
\end{tabular}

